\title{
Le syndrome du $X$ fragile : des mutations étonnamment ciblées et instables, et un gène à la recherche d'une fonction
}

Nous avions récemment rapporté dans médecine/sciences $\left(n^{\circ} 4\right.$, vol. 7, p. 378) la découverte d'une méthylation anormale de l'ADN, affectant un seul îlot $\mathrm{CpG}$ chez les mâles exprimant cliniquement et cytogénétiquement le syndrome du retard mental avec $X$ fragile [1, 2]. Nous avions pu cloner, en collaboration avec l'équipe de D. Cohen au CEPH (centre d'étude du polymorphisme humain), un segment d'ADN de 400 kilobases contenant cet îlot, sous forme de chromosome artificiel de levure (YAC) [3]. A partir de ces clones, nous avons isolé des sondes ADN permettant d'explorer directement l'îlot $\mathrm{CpG}$ anormalement méthylé. Nous avons ainsi découvert des anomalies étonnantes, non plus liées seulement à la méthylation (modification secondaire de l'ADN), mais à la présence même des mutations [4].

Ces mutations sont des réarrangements très localisés dont la cible est un petit fragment d'ADN (de 500 nucléotides) contenant l'îlot $\mathrm{CpG}$, et qui sont aisément détectés par la technique de Southern blot. Les mâles normaux transmetteurs ont une "insertion" de 150 à 500 nucléotides, qui est transmise soit inchangée, soit avec de petites variations de longueur, à leurs filles. Une extraordinaire instabilité des séquences d'ADN survient, avec une haute probabilité ( $80 \%$ dans nos observations), lorsque le chromosome muté est transmis à la génération suivante. En effet, les sujets avec chromosome $\mathrm{X}$ fragile détectable cytogénétiquement (hommes ou femmes) montrent des fragments de taille très augmentée (1000 à 3000 nucléotides) qui diffèrent à l'intérieur d'une même fratrie. On détecte en général une hétérogénéité dans le génome de ces patients (présence de plusieurs fragments de taille anormale) qui traduit l'existence de mutations somatiques additionnelles. Des observations très similaires ont été réalisées par l'équipe australienne de Grant Sutherland qui avait également isolé un clone YAC de la région, en collaboration avec l'équipe américaine de D. Schlessinger [5]. (Rappelons que Sutherland est un spécialiste incontesté de la maladie, qui avait découvert en 1977 les modalités d'induction du site fragile).

Nous avons étudié dans près de 50 familles la corrélation entre type de mutation et méthylation. L'allèle muté correspondant à la "petite insertion " apparaît non méthylé chez les mâles normaux transmetteurs et méthylé seulement sur le $\mathrm{X}$ inactif chez leurs filles. L'allèle porteur de la "grande insertion " est méthylé en général complètement chez les patients avec expression du site $\mathrm{X}$ fragile. Nous avons observé, dans de rares cas, des mâles qui apparaissent " mosaïque " pour une " petite insertion " non méthylée et une "grande insertion " méthylée. La région affectée par la méthylation anormale s'étend sur au moins $2 \mathrm{~kb}$, de part et d'autre du segment cible des mutations. Il faut noter toutefois que quelques familles ne présentent aucune des anomalies décrites cidessus. Pour certaines d'entre elles, un taux faible et peu significatif de $\mathrm{X}$ fragile n'avait été observé que chez un seul garçon retardé, et nous pensons qu'il s'agit d'erreurs de diagnostic liées au bruit de fond de la technique cytogénétique. Dans d'autres familles où l'expression du site fragile est très forte, des résultats préliminaires suggèrent une hétérogénéité génétique, et il pourrait s'agit d'un autre site fragile très proche, pas nécessairement lié à un retard mental (J. Boué, M.A. Voelckel, communications personnelles).

L'expression du syndrome $\mathrm{X}$ fragile implique donc une mutation en deux étapes ainsi qu'une méthylation anormale très localisée. Ce mécanisme tout à fait unique semble expliquer les anomalies de ségrégation du syndrome [6]. Le fragment cible fait partie de l'îlot $\mathrm{CpG}$ et contient une trentaine de répétitions du motif CGG (motif méthylable). Les changements de taille traduisent probablement l'amplification de cette séquence, peut-être par un mécanisme de recombinaison type échange inégal de chromatides-sœurs. Nos observations réconcilient en quelque sorte l'hypothèse de Laird (rôle d'une empreinte génomique) [7] et des hypothèses antérieures proposant l'existence d'une prémutation chez les mâles normaux transmetteurs, pouvant se transformer en pleine mutation lorsqu'elle est transmise par une femme [8, 9]. Il reste à expliquer pourquoi ce passage de la prémutation à la mutation est spécifique de l'oogenèse. Un rôle de l'inactivation du chromosome $\mathrm{X}$ dans ce passage nous paraît très attractif, mais n'a pas encore été prouvé. On pourrait penser, en adaptant l'hypothèse de Laird, que la région amplifiée en raison de la prémutation et qui, chez la femme, est méthylée sur le chromosome $\mathrm{X}$ inactif, ne peut être déméthylée lors de la réactivation du $\mathrm{X}$ préalable à l'oogenèse (l'allongement de la séquence CGG entraînant une augmentation importante du nombre de sites méthylés). Une conformation anormale de la région amplifiée méthylée pourrait alors créer des difficultés de réplication, 
entraînant l'allongement de la séquence (CGG)n (on sait que la méthylation favorise la forme $Z$ pour un $A D N$ de structure (CG)n, et de manière anecdotique, on peut signaler que la région mutée apparaît particulièrement résistante à l'amplification par PCR). Le passage de la prémutation à la mutation ne se ferait, selon ce schéma (figure 1), que si la femme transmet la mutation sur le $X$ inactif (avec donc une probabilité de $50 \%$ ). En fait l'hypothèse de Laird prédisant que les mâles normaux transmetteurs doivent être aussi nombreux que les mâles atteints [7], il devient possible de tester directement cette prédiction, par l'analyse systématique et complète de tous les membres des familles avec $\mathrm{X}$ fragile. La contradiction avec la fréquence de $80 \%$ que nous avons observée pour la transition de la prémutation à la mutation n'est peut-être qu'apparente, car il existe un biais évident privilégiant l'étude des familles dont plusieurs membres expriment la maladie. Il reste toutefois possible que les anomalies de méthylation ne soient que la conséquence de la mutation, l'allongement important de la séquence CGG et l'augmentation des sites méthylables perturbant le contrôle normal de la méthylation (cela pourrait être plus compatible avec l'existence de mâles "mosaïques" cités plus haut).

Les îlots $\mathrm{CpG}$ marquent en général l'extrémité 5' de gènes. Il était donc très tentant de chercher un gène adjacent à l'îlot $\mathrm{CpG}$ directement impliqué dans le mécanisme de la maladie. Cela a été réalisé avec succès par une collaboration entre laboratoires américains (Caskey à Houston, TX; Warren à Atlanta, GE) et hollandais (Oostra à Rotterdam, Van Ommen à Leiden) et est rapporté dans un article signé par 21 auteurs et publié avec une grande célérité par Cell [10] (on peut noter que le $\mathrm{CEPH}$, bien que non représenté dans les auteurs, a joué un rôle important en mettant à disposition son excellente librairie de YAC). Un ADNc a été cloné correspondant à un ARNm de 4,8 kb exprimé (notamment) dans le cerveau et les lymphocytes, et dont l'extrémité 5' paraît correspondre à l'îlot $\mathrm{CpG}$. Ce

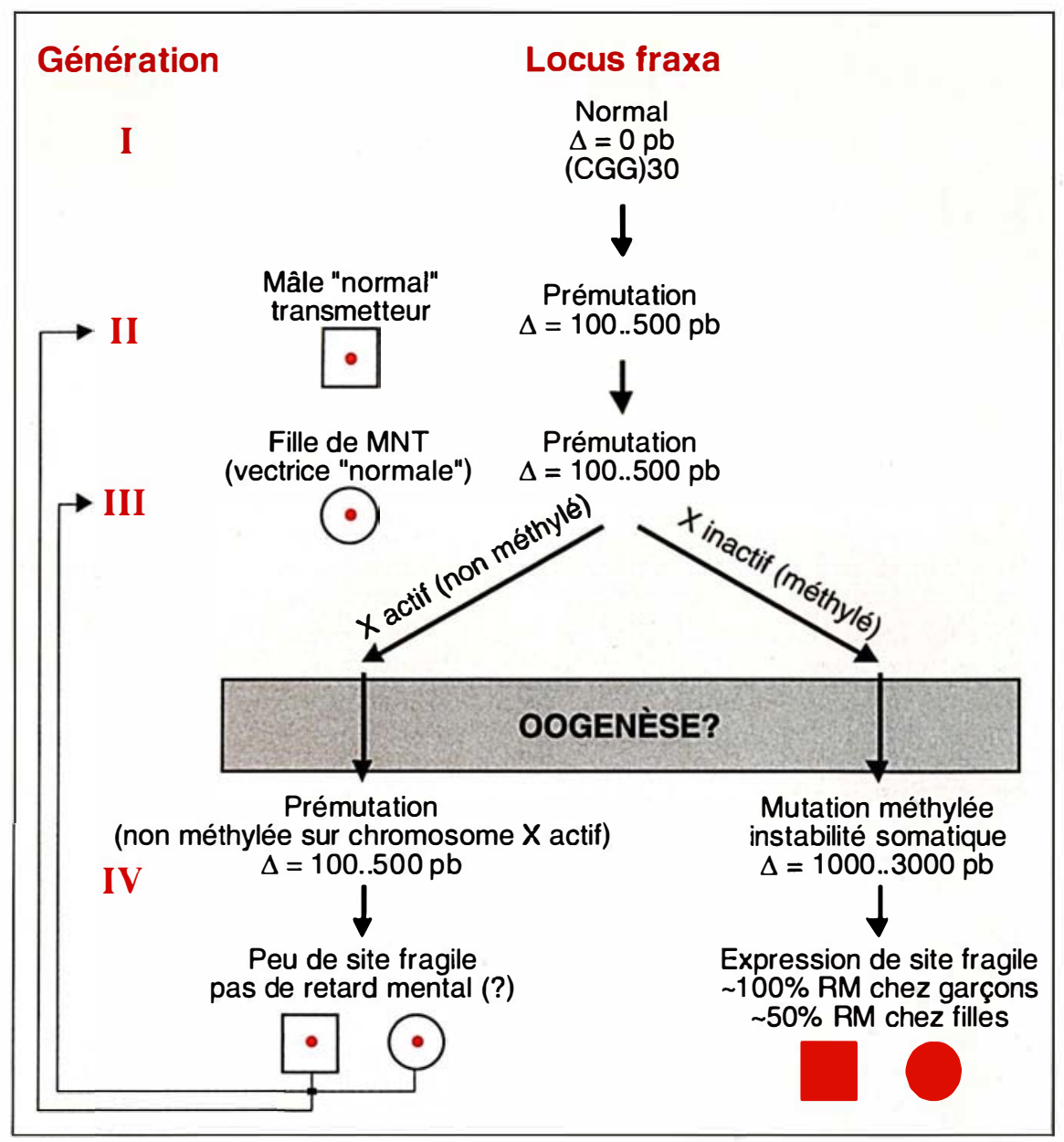

Figure 1. Schéma hypothétique du rôle de l'inactivation du chromosome $X$ dans le passage de la prémutation à la mutation. L'allongement du fragment cible ( $\Delta$ ) est mesuré en paire de bases. II n'a pas été formellement démontré que cet allongement résulte de l'amplification de la séquence (CGG)n. La prémutation pourrait a priori apparaître dans l'un ou l'autre sexe, et le passage direct d'un allèle normal à la mutation complète reste possible (mais n'a pas été observé jusqu'ici). L'expression du site fragile serait due à la grande difficulté à répliquer la région très allongée et méthylée. Le retard mental (RM) et les anomalies morphologiques pourraient être expliqués par une inhibition, soit de l'initiation de la transcription du ou des gènes adjacents (rôle de la méthylation), soit de l'élongation de la transcription, soit encore de la traduction (amplification de la séquence CGG). Ce schéma simplifié (simpliste ?) ne tient pas compte de certains cas rares (mâle " mosaïque " pour la prémutation et la mutation, réversion possible de la mutation vers la prémutation). MNT = mâle normal transmetteur.

et pourrait donc en principe coder pour une protéine comportant, chez un sujet normal, une trentaine d'arginines à l'extrémité N-terminale (toutefois cette protéine n'a pas été encore identifiée et une initiation à un AUG plus distal ou une maturation enlevant ce domaine sont donc possibles). Un allongement de la séquence (CGG)n chez les patients pourrait inhiber la traduction ou déstabiliser l'ARN messager. Une première analyse ne montre pas d'homologie frappante entre cette protéine putative et des protéines de fonction connue. D'autre part, 
l'analyse de l'expression de ce gène chez des sujets atteints n'a pas encore été réalisée. Rappelons que le phénotype $\mathrm{X}$ fragile comportant des anomalies morphologiques (faciès, macro-orchidie), on s'attend à ce que le (ou les) gène(s) impliqués s'expriment dans d'autres organes que le cerveau. Il reste d'ailleurs possible qu'un deuxième gène soit présent de l'autre côté de l'îlot CpG. L'identification de la fonction du ou des gènes dont l'expression est pertubée chez les patients devrait apporter des éléments fondamentaux pour la compréhension des mécanismes pathologiques, en particulier du retard mental.

Une différence importante est à noter entre le syndrome du $\mathrm{X}$ fragile et la plupart des autres maladies génétiques connues. En général, la cible des mutations comprend la totalité du gène (exons, séquences nécessaires pour l'épissage ou séquences régulatrices). Dans le cas du $\mathrm{X}$ fragile, la cible de la mutation est une région très petite, qui se trouve être à l'extrémité 5' d'un gène. Ce segment est peut-être le point de mutation le plus chaud du génome humain. En effet, pour expliquer la fréquence de la maladie, on est en principe obligé de supposer un taux de néomutation au moins égal à celui de la myopathie de Duchenne (dont le gène s'étend sur 2-3 millions de paires de bases) [11]. Toutefois, il faut noter qu'aucune néomutation prouvée n'a encore été observée dans une famille avec $X$ fragile, et si la fréquence des mâles normaux transmetteurs était effectivement égale à celle des patients, cela aurait pour conséquence de faire baisser l'estimation du taux de néo-mutation*.

\footnotetext{
- D'après Haldane, le taux de mutation pour une maladie liée au chromosome $X$ est égal à (1-f)x, ou $f$ est la fertilité relative (comparée à un mâle normal) d'un mâle porteur de la mutation et $x$ l'incidence de la maladie chez les mâles. Dans le cas du syndrome $X$ fragile, $f$ serait au moins égal à la proportion de mâles normaux transmetteurs, car ceux-ci ont une fertilité normale. L'absence de néomutation prouvée (mâle atteint dont la mère n'est pas vectrice) pourrait indiquer qu'il n'y a pas (ou très rarement) passage direct d'une séquence normale à la mutation complète.
}

$\mathrm{m} / \mathrm{s} n^{\circ} 6$, vol. 7 , juin-juillet 91

\section{Perspectives diagnostiques} Il est maintenant possible de détecter très simplement, au niveau de l'ADN, les anomalies génétiques responsables du syndrome $\mathrm{X}$ fragile (quelque soit le sexe ou l'expression clinique), par une analyse en Southern blot nécessitant l'utilisation d'une seule sonde et d'une seule enzyme de restriction (par exemple EcoRI). Cela devrait bouleverser le diagnostic et le conseil génétique de cette maladie. En effet, la lourdeur de l'analyse cytogénétique faisait que l'indication de recherche du site fragile était souvent limitée aux mâles retardés mentaux ayant des antécédents familiaux (frères, cousins ou oncles maternels) évocateurs d'un problème génétique lié au chromosome $\mathrm{X}$. Nous proposons que l'analyse de l'ADN soit maintenant réalisée en première intention chez un garçón ou une fille avec un retard mental inexpliqué (ou un comportement autistique). Un diagnostic précoce est en effet nécessaire pour mettre en œuvre un conseil génétique approprié. En fait, le syndrome du X fragile devrait être la première maladie génétique où l'analyse de l'ADN est l'élément diagnostic principal. L'analyse de l'ADN devrait également résoudre les problèmes du dépistage des vectrices et du diagnostic prénatal, dans les familles à risque. La différenciation entre " prémutation " entraînant peu ou pas de signes cliniques et la mutation, devrait permettre d'affiner le diagnostic prénatal sans toutefois, pour les filles, permettre une prédiction complète, car l'expression clinique dépendra sans doute de la proportion du chromosome muté qui est porté par le chromosome $\mathrm{X}$ actif, dans les organes ou cellules (non connues à l'heure actuelle) impliqués dans les manifestations pathologiques. L'identification de garçons porteurs de la prémutation pourrait poser des problèmes éthiques. Le risque pour leur descendance n'apparaîtra que quand ils seront grands-pères. On peut donc se poser le problème de l'utilité à l'heure actuelle d'un tel diagnostic. La recherche systématique des mâles normaux transmetteurs dans les familles à risque est toutefois d'un intérêt fondamental pour la compréhension des mécanismes de la maladie.
1. Oberlé I, Mandel JL. Retard mental avec $\mathrm{X}$ fragile : une empreinte génomique très localisée, étroitement liée à l'expression clinique. médecine/sciences 1991; 7 : 378-9.

2. Vincent A, Heitz D, Petit C, Kretz C, Oberlé I, Mandel JL. Abnormal pattern detected in fragile $X$ patients by pulsed field gel electrophoresis. Nature 1991 ; 349 : 624-6. 3. Heitz D, Rousseau F, Devys D, et al. Isolation of normal sequences that span the fragile $\mathrm{X}$ site and identification of a $\mathrm{CpG}$ island involved in fragile $X$ expression. Science 1991 ; 261 : 1236-9.

4. Oberlé I, Rousseau F, Heitz D, et al. Instability of a 550 bp DNA segment and abnormal methylation in fragile $\mathrm{X}$ syndrome. Science 1991 ; 252 : 1097-102.

5. Yu S, Pritchard M, Kremer E, et al. Fragile $\mathrm{X}$ genotype characterized by an unstable region of DNA. Science 1991; 252 : 1179-81. 6. Jordan BR, Mattei JF. Retard mental lié à la fragilité du chromosome $\mathrm{X}$ : où en est- on en 1989 ? médecine/sicences $1989 ; 5: 450-8$. 7. Laird CD. Proposed mechanism of inheritance and expression of the human fragile $\mathrm{X}$ syndrome of mental retardation. Genetics 1987 ; 117 : 587-99.

8. Pembrey ME, Winter RM, Davies KE. A premutation that generates a defect at crossing over explains the inheritance of fragile $\mathrm{X}$ mental retardation. Am J Med Genet 1985; 21 : 709-17

9. Nussbaum RL, Ledbetter DH. Fragile $X$ syndrome : a unique mutation in man. Ann Rev Genet 1986; 20 : 109-45.

10. Verkerk AJMH, Pieretti M, Sutcliffe M, et al. Identification of a gene (FMR-1) containing a CGG repeat coincident with a breakpoint cluster region exhibiting length variation in fragile X syndrome. Cell 1991, 65, 905-14. 11. Vogel F, Crusio WE, Kovac C, Fryns JP, Freund M. Selective advantage of $\mathrm{fra}(\mathrm{X})$ heterozygotes. Hum Genet $1990 ; 86$ : 25-32. 